\title{
FRIEDRICH LUDWIG VON MAYDELLS BRIEF AUS ROM VOM JAHR 1823
}

Es könnte vermutet werden, dass über Friedrich Ludwig von Maydell (1795-1846) - „einen der hervorstehendsten Vertreter der Romantik in den baltischen Ländern"1 - in Hülle und Fülle geschrieben worden ist, angefangen mit den umfangreichen Schriften Wilhelm Neumanns aus der Zeit der Wende vom 19. zum 20. Jahrhundert ${ }^{2}$ bis hin zu der kürzlich erfolgten, von seinen „Fünfzig Bildern aus der Geschichte der deutschen Ostsee-Provinzen Russlands" angeregten Ausstellung und den entsprechenden Publikationen in den Jahren 2013-2015. ${ }^{3}$ Dennoch war die Rekonstruktion von Maydells Lebensgeschichte schwierig. Als Hauptquellen verbleiben Neumanns Biografien, doch die von ihm genutzten Materialien sind unbekannt. Von Maydells römische Periode erreichte Neumann und uns vor allem dank der Erinnerungen seines Freundes Adrian Ludwig Richters (1803-1884) und von Maydells Name trifft man in den Memoiren und Biografien anderer Deutschrömer (Wilhelm von Kügelgen, Julius Schnorr von Carolsfeld, Richard Rothe u. a.), ${ }^{4}$ doch diese

DOI: http://dx.doi.org/10.12697/BJAH.2016.12.06

Übersetzung aus dem Estnischen von Marju und Olaf Mertelsmann.

1 Voldemar Vaga, „Maydell, Friedrich Ludwig von”, Eesti kunsti ja arhitektuuri biograafiline leksikon (Tallinn: Eesti entsüklopeediakirjastus, 1996), 303-304.

2 Wilhelm Neumann, Friedrich Ludwig v. Maydell. Ein baltischer Maler (Riga: Druck von W. F. Häcker, 1897); Wilhelm Neumann, Ein Künstlerlos. - Aus alter Zeit. Kunst- und kulturgeschichtliche Miszellen aus Livland, Estland und Kurland (Riga: Verlag von G. Löffler, 1913), 109-126 u. a.

3 Friedrich Ludwig von Maydelli pildid Baltimaade ajaloost = Friedrich Ludwig von Maydells Baltische Geschichte in Bildern = Friedrich Ludwig von Maydell's Baltic history in image, hrsg. von Linda Kaljundi, Tiina-Mall Kreem (Tallinn: Eesti Kunstimuuseum, 2013); Kunstnik ja Kleio. Ajalugu ja kunst 19. sajandil = The artist and Clio. History and art in the 19th century = Der Künstler und Klio. Geschichte und Kunst im 19, hrsg. von Tiina-Mall Kreem, Eesti Kunstimuuseumi Toimetised, 5 [10] (2015). 4 Siehe ausführlicher: Friedrich Noack, Das Deutschtum in Rom seit dem Ausgang des Mittelalters, Bd. 2 (Stuttgart [etc.]: Deutsche Verlags-Anstalt, 1927), 388. 
sind alle aus der Perspektive von Außenstehenden verfasst und von ihrem Charakter her willkürlich, damit bleiben sie fragmentarisch. Umso wertvoller sind unerwartet ans Tageslicht gekommene Archivquellen, die vom Künstler mit eigener Hand geschrieben wurden, welche helfen, die „Gedächtnislücken” der späteren Rezeption zu füllen.

Zwei im Estnischen Historischen Archiv im Bestand des Familienarchivs der von Ungern-Sternbergs erhaltenen Briefe ${ }^{5}$ schrieb von Maydell in den Jahren 1822-1823 an seinen Onkel, den damaligen Syndikus der Universität Dorpat (Tartu), Otto Christian Sigismund von Ungern-Sternberg (1778-1861). In Dorpat hatte von Maydell in den Jahren von 1820 bis 1822 als Archivar in der seinem Onkel unterstellten Kanzlei gearbeitet ${ }^{6}$ und Vorlesungen an der Juristischen Fakultät gehört. Seine Lehrkräfte lobten ihn und auch Rektor Gustav Ewers war mit seiner Arbeit bei der Ordnung des Universitätsarchivs zufrieden. ${ }^{7}$ Zur selben Zeit nahm von Maydell Zeichenunterricht bei Karl August Senff und im Jahr 1821 besuchte er auch die Vorlesungen zur Kunstgeschichte von Prof. Karl Morgenstern. ${ }^{8}$ Im selben Herbst fällte von Maydell die grundlegende Entscheidung, im Ausland Malerei zu erlernen. Im Februar 1822 begann er die Reise nach Italien. ${ }^{9}$ Noch davor, im Januar 1822, hatte er sich mit Therese von Müller (1795-1882) verlobt, die in der Heimat blieb und auf seine Rückkehr wartete.

Der erste, im Archivbestand der von Ungern-Sternbergs erhaltene Brief wurde am 20. Juli 1822 in Stonsdorf in Niederschlesien (heute Staniszów) verfasst und enthält hauptsächlich Familiennachrichten (von Maydell hatte gerade seine in einer Bildungsanstalt der Herrnhuter in Großhennersdorf lernenden Cousins besucht). Doch es ergibt sich, dass

5 Eesti Ajalooarhiiv / Estnisches Historisches Archiv [EAA], 1423-1-26, Schreiben von Angehörigen, Verwandten und Bekannten von Otto Christian Sigismund von Ungern-Sternberg.

Dasfreiherrliche Geschlecht von Maydell: Für die Familie zusammengestellt und in Druck gegeben von Baron Karl von Maydell (Helsingfors: Druckerei der finnischen Litteraturgesellschaft, 1868), 293 Tartu un [Karl [Karl Morgenstern], Subscriptionstisten zu Morgensterns Vorlesungen in Dorpat 1803-1836.

Dorothea (Doris) von Ungern-Sternberg 1787-1828: Ein Lebensbild, nach Briefen und andere Unterlagen, zusammengestellt von Heinrich Seesemann, Baltische Erinnerungen und Biografien, Bd 4 (Hannover-Dohren. Verlag Harro v. Hirschheydt, 1979), 223. - Aus den Familienerinnerungen in voutschland und Italien Malerei zu studieren, bereits bevor er in Dorpat ankam, dusch thatte, Tod des Braters A f 1818 entshed sich,

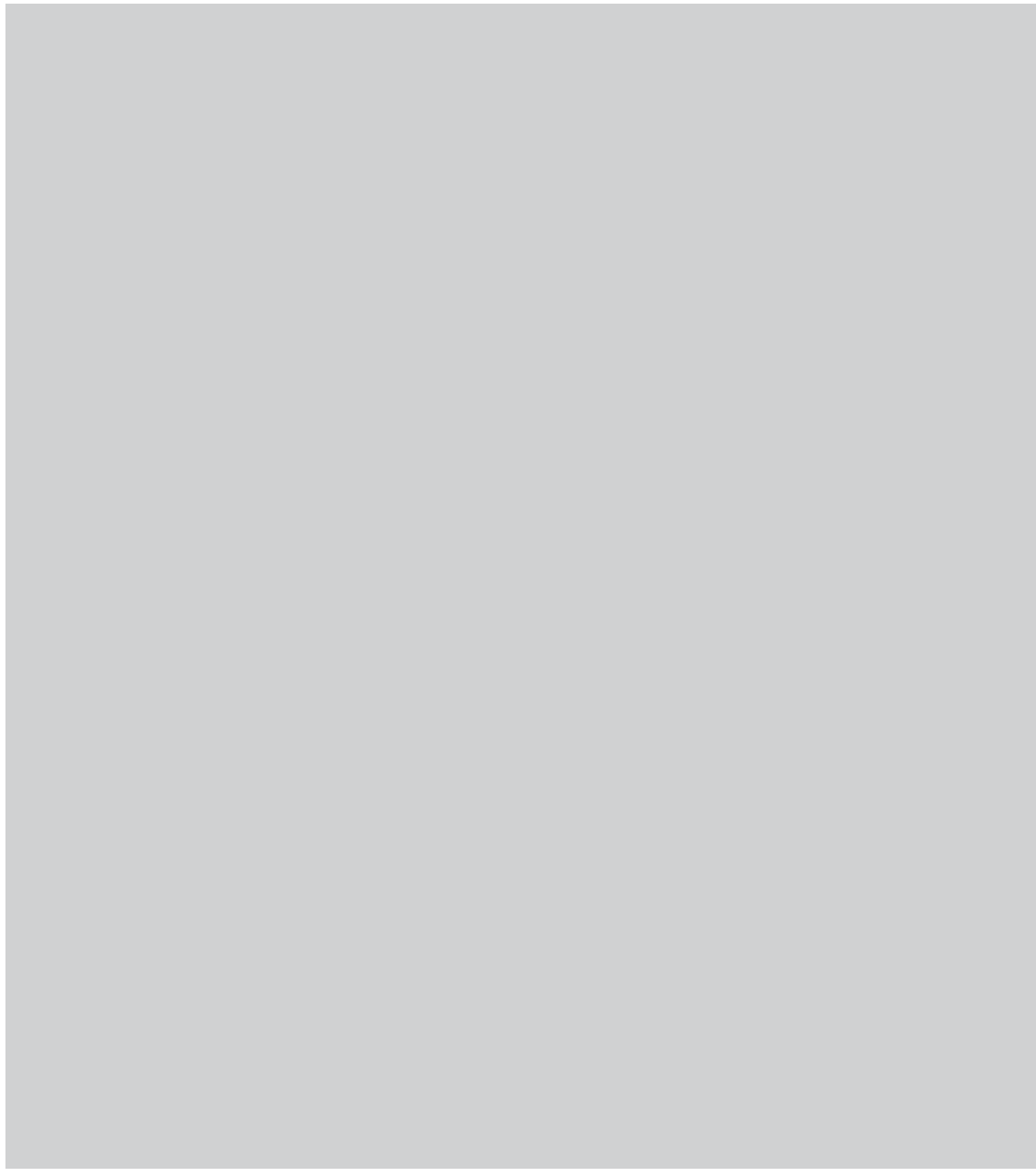

Abb. 1. Friedrich Ludwig von Maydell, Selbstbildnis, Aquarell, 1827. Estnisches Kunstmuseum.

von Maydell vor der Ankunft in Italien in Prag und Basel vorbeischauen wollte.

Der zweite, hier veröffentlichte Brief hilft aber auf beste Weise, von Maydells künstlerische Biografie zu präzisieren und zu ergänzen. Dies beginnt schon mit der ersten Zeile: Obwohl aus mehreren Quellen bekannt ist, dass von Maydell sich bereits im Jahr 1823 in Rom aufhielt, ${ }^{10}$

10 Noack, Das Deutschtum in Rom seit dem Ausgang des Mittelalters, 388 
hält sich die um ein Jahr spätere Datierung Neumanns in der Literatur hartnäckig. Das im Kopf dieses Briefes befindliche Datum „Rom. den 30 Jan. 1823." bestätigt, dass sich von Maydell bereits zu Beginn des Jahres 1823 in Rom eingerichtet hatte und möglicherweise schon am Ende des Vorjahres eingetroffen sein könnte. Damit kann in diesem Licht die Behauptung nicht mehr ernst genommen werden, dass von Maydell für ein Jahr in Stuttgart an der Karlsschule studiert hätte. ${ }^{11}$

Ein zweiter und wichtigerer Umstand, der im Brief auftritt, ist mit dem Namen Johann Friedrich Overbeck (1789-1869) verknüpft, den von Maydell im Schreiben als seinen Lehrer bezeichnet und unter dessen Anleitung er in Rom zumindest in der Anfangszeit arbeitete. Und wieder - obwohl Overbeck in ihren Erinnerungen von Maydells Witwe genannt wird ${ }^{12}$ und auch Overbecks Biografin Margaret Howitt von Maydells Namen unter seinen Schülern erwähnt, ${ }^{13}$ gilt Overbecks Einfluss auf von Maydell bis jetzt eher als indirekt. Doch wie sich aus dem Schreiben ergibt, war es gerade Overbeck als geistiger Anführer der Nazarener und als Person - mit von Maydell, der einen herrnhutischen Hintergrund hatte, offensichtlich sympathisierend - dessen Gunst ausschlaggebend war, warum von Maydell in Rom schließlich den Lebensweg eines Künstlers wählte.

Overbeck hatte zu diesem Zeitpunkt schon den Status einer lebenden Legende eingenommen. Er war der einzige unter den Nazarenern der ersten Generation, der in Rom geblieben war, während zur selben Zeit sich die anderen wieder zurück in ihre Heimat bewegten. Als Begründer der Gruppe war er selbst der ernsthafteste Vertreter ihrer Ideen, angefangen von seinem äußeren Eindruck und seinem Lebensstil und endend mit seinem vom Spätmittelalter und der Frührenaissance, vor allem von Fra Angelico und Raffael inspirierten Schaffen. Als charismatische Persönlichkeit sammelten sich um ihn herum Unterstützer und Schüler und mit seiner einfachen sowie anspruchslosen Erscheinung erweckte er auch bei jenen Hochachtung, deren Ansichten mit den seinen nicht in allem übereinstimmten. ${ }^{14}$

11 Das freiherrliche Geschlecht von Maydell, 291.

2 Ibidem, 293.

3 Margaret Howitt, Friedrich Overbeck: Sein Leben und Schaffen, Bd. 2 (Freiburg in Breisgau: Herder'sche Verlagshandlung, 1886), 105

4 Zu Overbecks Imago des Mönch-Künstlers siehe: Mitchell Benjamin Frank, German Romantic Painting Redefined: Nazarene Tradition and the Narratives of Romanticism (Aldershot; Burlington, VT: Ashgate, 2001).
Zu Overbeck waren zuvor in den Jahren 1817-1819 auch Kontakte von „dreier estländischer Maler”, Otto Ignatius, Gustav Hippius und August Pezold, entstanden, ${ }^{15}$ von denen Ignatius, der sich religiösen Motiven widmete, für denjenigen gehalten wird, der am stärksten von Overbeck beeinflusst wurde. ${ }^{16}$ Wenn wir in Leopold Pezolds mehrere Jahrzehnte später verfassten Reisebeschreibung lesen können, wie Hippius und der kurländische Künstler Johann Leberecht Eggink sich in Rom mit einem Hauptwerk der Nazarener bekannt machten, mit einem nach Motiven der Josefsgeschichte im Alten Testament anzufertigenden Fresko im Palazzo Zuccari (der sogenannten Villa Bartholdy; 1816-1817), dann befand sich Overbeck bei der Ankunft von Maydells in Rom gerade in der Mitte des Malens eines Freskos nach Motiven von Torquato Tassos „Das befreite Jerusalem“ im Casino Massimo (1817-1828). Der Einfluss dieses Freskenzyklus auf von Maydells zwanzig Jahre später erschienene „Fünfzig Bilder..." ist offensichtlich. ${ }^{17}$

So wie die Romantiker in der Malerei kein bestimmten Regeln unterworfenes technisches Wissen sahen, denn dies war eine Weise, sich selbst auszudrücken und sich selbst zu bestimmen, ${ }^{18}$ so eignete sich von Maydell in Rom nicht so sehr neue Fertigkeiten an, als dass er sich vielmehr selbst fand. Zusätzlich zu neuen Fakten stellt der vorliegende Brief ein charakteristisches Beispiel für den mühevollen Weg, ein Künstler zu werden, dar, die Suche nach sich selbst und das Finden eines Kompromisses zwischen der inneren Berufung und den Erwartungen von Familie und Gesellschaft. Sowohl von Maydells Vater, sein Onkel und eine ganze Reihe anderer Verwandter verfügten über eine juristische Ausbildung. Gleichzeitig war von Maydell keinesfalls der erste in seinem Verwandtenkreis, der von diesem Weg abwich: der in Reval (Tallinn) lebende Sohn seines Großonkels, eines Juristen, Johann Karl Emanuel von Ungern-Sternberg (1773-1830) hatte eine vergleichbare Entscheidung ungefähr 15 Jahre zuvor getätigt, er begann 1806 in Dresden Kunst zu studieren, setzte später dennoch auch die Arbeit als Beamter der Ritterschaft fort.

15 Leopold Pezold, Kolme Eestimaa kunstniku rännuaastad, übers. von Anne Lõugas (Tallinn: Kunst, 1994), 58.

Wilhelm Neumann, Lexikon baltischer Künstler (Riga: Verlag von Jonck \& Poliewsky, 1908), 75 Anne Untera, „Pühendumus. Friedrich Ludwig von Maydelli elu ja loomingu sisulisest teljest Kunstnik ja Kleio. Ajalugu ja kunst 19. sajandil, 129-130; Anu Allikvee, „Friedrich Ludwig von Maydel 
Von Maydells Lehrjahre in Rom und in Italien dauerten bis zum Jahr 1827 an. In die Heimat zurückgekehrt, fand sein Talent in den folgenden Jahrzehnten sehr unterschiedliche Ausdrucksformen, weshalb die ihm nahestehenden Menschen und auch die nachfolgenden Generationen wohl kaum einen Grund hatten, an der Korrektheit seines Anfang 1823 in Rom gewählten Wegs zu zweifeln.

Rom. den 30. Jan. 1823. ${ }^{19}$

Lieber Onkel, ${ }^{20}$

Ein lebhaftes Andenken an Sie, das heute besonders in mir erweckt wurde, läßt mich nicht eher ruhen, als bis ich Papier u. Feder ergriffen, in Gedanken den Berg zu Ihrer Wohnung hinaufgegangen $\mathrm{u}$. Sie besucht habe. ${ }^{21}$ Aus eigener Erfahrung werden Sie ja wissen, wie wunderbar lebendig solche heimische Bilder oft in der wilden Fremde werden $\mathrm{u}$. wie sie dann sogar lieb u. wohlthuend sind. Hier nun vollends, wo Alles, alles so ganz anders als bey uns, sowohl der außern Form, als dem innern Sinn nach, besonders in Sitte $\mathrm{u}$. Lebensweise, deren kleine Eigenheiten in heimisches Wesen mir bey uns so recht gefielen, hier mahnt es mich oft schmerzlich an, das liebe Vaterland, das ich immer lieber gewinne, je mehr andere Länder ich sehe, $u$. wenn es nur irgend möglich ist, gegen keins vertauschen will. Es fällt mir sonst wohl nicht ein, über meinen jetzigen Wohnort zu klagen, ich finde ihn vielmehr herrlich $u$. für meinen gegenwärtigen Zweck grade passend, Beschäftigung des Geistes, Arbeit u. Muße dazu voll auf, aber das Herz bleibt eben kalt, u. die rechte Erquickung nach d. Arbeit fehlt, wenn ich nicht eben glücklicherweise so reich an Errinnerungen aus der Heimath wäre, u. mich d. Hoffnung, einst so vieles schöne Glück dort wiederzufinden, noch reicher machte. Geduld, Geduld muß ich mir indeß dabey oft zurufen, für die lange Zeit der Entbehrung, die das ernste Studium fordert. Ja, guter Onkel, ich bin wohl so fest als man es menschlicherweise seyn kann, entschlossen Maler zu werden u. zu bleiben. Der Kampf

19 EAA, 1423-1-26. Handschrift transkribiert: Moonika Teemus, Mare Rand.

20 Otto Christian Sigismund von Ungern-Sternberg (1778-1861), der Bruder von Maydells Mutter, studierte in Göttingen Jura, war Syndikus der Universität Dorpat im Zeitraum 1801-1812, danach i Riga am Livlandischen Hofgericht als Assessor tätig, 1819-1826 war er erneut Syndikus.

Stadto um diesen Entschluss hat Monate lang gedauert, u. doch überließ ich zuletzt die Entscheidung Andern, da mir bey der brennenden Lust, nicht Besonnenheit $\mathrm{u}$. richtige Beurtheilung zutraute. Das fortwährende Hin u. Herbewegen des einen Gedankens, auf der langen einsamen Reise hieher, wo das zweifelhafte Urtheil meiner wartete, hatte mich zuletzt so ängstlich $\mathrm{u}$. besorgt gemacht, daß ich bey meiner Ankunft hier beynahe schon entschlossen war, nach einem Aufenthalt von ein paar Monaten, wieder nach Hause zu kehren. Hier aber, erzähle Ihnen aufrichtig Alles, lieber Onkel, als ich die Meisterwerke der alten Kunst, u. manchen herrlichen neuen Meister sah, wuchs mir der Muth von Tag $z u$ Tage. Ich ging zu Overbeck ${ }^{22} u$. bat ihn mich zu seinem Schüler aufzunehmen, was er freundlich that, $\mathrm{u}$. fing nun an zu malen, Anfangs nach Gyps, Grau in Grau, u. dann ein altes Bild, woran ich noch arbeite Einige Compositionen, die mich selbst überraschten, [S. 2] da ich mir es noch gar nicht bewust geworden war, was ich in der Zeit d[urc]h das viele Sehen alles gelernt hatte, gefielen auch dem Meister sehr wohl, $\mathrm{u}$ er fand es unbegreiflich, wie jemand der fast so ganz sich selbst überlassen gewesen, so richtig $\mathrm{u}$. bestimmt zeichnen könne. So ging ich denn nun endlich einmal zu ihm hin, u. fragte ihn, ob er glaube daß ich vor Gott, mir, $u$. andern Menschen verantworten könne, wenn ich Maler würde, u. einen andern Wirkungskreis, wo ich nicht unnütz zu seyn hoffe, verließe. Er antwortete nach einigem Nachdenken, daß er, was Gabe $u$. Talent beträfe, es auf sein Gewissen nähme, mir nach seiner Ueberzeugung zu versichern, daß ich den schweren Künstlerweg einzuschlagen wagen dürfe, wenn ich nur selbst meiner Ausdauer bey der oft trockenen Arbeit u. bey der einmal gefaßten Idee, sicher sey. Da letzteres nun mit Gottes Hülfe d[urc]h eigener Kraft d[urc]h zuführen ist, wenn nur d. nöthigen Gaben nicht fehlen, so stand mein Entschluß wohl gleich fest, so schwer es mir auch im Anfang ist, u. noch seyn wird, mit langsamen festen Schritten das Feld zu d[urc]h ziehen, auf welchem ich sonst nach Lust $\mathrm{u}$. Laune von allen Seiten mein Streifparthien gemacht habe. Ich fühle wohl gar zu deutlich daß es gehen wird, so große Hindernisse auch noch zu übersteigen sind. Hier bin ich in meinem

22 Friedrich Overbeck (1789-1869), deutscher Maler, war einer der Begründer und Hauptideologen der Künstlergruppe der Nazarener. 
rechten Element, wie ein Fisch im Wasser, ${ }^{23}$ ich arbeite von Morgen bis Abend, aber jeder Zug der Hand ist ein Schritt vorwärts zu dem Ziele nach welchem alle meine Gedanken streben. Solch ein Gefühl habe ich noch gar nicht gekannt, so ganz u. gar einig u. ungespalten mit einem Tichten $\mathrm{u}$. Trachten zu seyn, $\mathrm{u}$. nichts zu thun, wobey ich nicht von ganzem Herzen dabey wäre. Das, lieber Onkel ist doch wohl Beruf, dem ich folgen darf. Mich interessiert $u$. freut hier Nichts als meine Kunst, $u$. alles was mir auch nur eine Stunde kostet, wird auf d. Seite geschoben. Ich besuche keine Gesellschaften, mache keine Spatziergänge, besuche keine Merkwürdigkeiten, ausser wo ich Gründen, $\mathrm{u}$. das ungern, nachgeben muß. So arbeite ich denn auch mit Freuden drauflos, sicher vertrauend, der liebe Gott, werde mir den Weg auf welchen er mich auf so wunderbare Weise geleitet hat, auch glücklich zurücklegen helfen, u. mich hinführen, wo es für mich $u$. Andre am besten seyn wird. Auf ein brillantes Glück darf in jetziger Zeit nicht hoffen, aber alle meine Wünsche sind auch erreicht wenn ich nur vor drückenden Nahrungssorgen mich sichern kann, da ich ja das Glück das Alles übrige ersetzt, in mir trage, $u$. meine liebe Braut ${ }^{24}$ genug kenne um zu wissen, daß auch sie dann glücklich $u$. zufrieden seyn wird. Ich bin um so getroster, da ich mir sicher bewust bin, daß ich ohne Zaudern aufgegeben hätte, was mir als das größte Glück vorschwebt u. umgekehrt wäre, wenn das Urtheil meines [S. 3] Meisters anders ausgefallen wäre. So finde ich es aber an Allem was ich hier um mich sehe bestätigt, u. könnte ganz schwindlich werden, wenn ich wörtlich glauben wollte, was mir schon von mehreren Seiten versichert worden ist. Glücklicherweise steht aber mein Ziel so hoch $u$. herrlich vor mir, daß ich gar zu gut fühle wie weit ich noch davon entfernt bin, u. es mir nicht einfällt mich an andern Künstlern erst messen zu wollen. Dazu habe ich es auch noch so schön mit meinem Meister getroffen, der so einzig unter allen hiesigen Künstlern dasteht; sowohl in seiner Kunst als auch als Mensch, daß alle noch lange an ihm zu lernen haben. Seine Bilder üben die anspruchslose stille Gewalt, von der man gar nicht los kommen kann, wie er selbst.

23 Die Äußerung „Der Fisch gehört ins Wasser, der Künstler nach Rom!” wird wohl dem von 1822 bi 1830 in Rom tätigen, deutschen Maler und Grafiker Bonaventura Genelli (1798-1868) zugeschrieben, doch sie könnte auch weiter verbreitet gewesen sein

24 Therese Agrippina Constanze von Müller (1795-1882), von Maydells Verlobte, die Tochter seiner Tante.
Sie wissen daß er u. Cornelius ${ }^{25}$ für die Häupter der neu-altdeutschen Schule gehalten werden, ${ }^{26}$ aber bey da sind, jeder nach seiner individuellen Weise, so weit von der Carricatur der alten Schule, $\mathrm{d}[$ urc]h die sich manche Neue auszeichnen wollen, entfernt, daß dieser Name in der Bedeutung, wie man ihn gewöhnlich versteht, gar nicht auf sie paßt. Seine Gestalten sind so rein Raphaelisch, ${ }^{27}$ so weit entfernt von theatralischem Pathos, als von verkrüppelter Naivität, seine Composition aber so klar u. einfach $\mathrm{u}$. doch reich, daß man gar nicht satt dran sehen kann, u. einem das Herz recht aufgeht. Das haben selbst Franzosen, die ganz in ihre Manier versessen waren, eingestehen müssen. ${ }^{28}$ Bey solch einem Meister ist gut seyn. Mit noch einem Mitschüler arbeite ich in einem Nebenzimmer bey ihm, u. alle Abend vor dem Nachhausegehen, beschließen wir das Tagewerk indem wir besehen, was er den Tag über geschafft hat $u$. plaudern noch ein halbes Stunden mit ihm. ${ }^{29}$ Er ist dabey so bescheidener, einfacher Art, daß wir uns so zusammen sähe, ohne uns genauer zu kennen, nie errathen würde, daß er der Meister $\mathrm{u}$. wir d. Schüler wären. Die Meinungen werden so frey geäußert, u. er prüft unsere Bemerkungen so genau, als wenn wir auf gleichem Brette stünden. Mein Mitschüler, der im Malen schon viel weiter als ich ist, u. auch sonst ein tüchtiges Talent hat, ist ein braver Junge, der mit noch einem Architeckten $u$. einem Bildhauer, fast meinen einzigen Umgang ausmacht, indem ich zu mehr, keine Zeit übrig habe. Wir bringen gewöhnlich d. Abende miteinander zu, wo bey einem Glas Wein etwas kalten Küche, u. der gemüthlichen Pfeife, wechselsweise Architectur, Perspective u.

25 Peter von Cornelius (1783-1867), deutscher Maler, war einer der bekanntesten Vertreter der Nazarener, verließ zum Jahr 1823 Rom. Overbecks und Cornelius' weitere Bekanntheit in Deutschlan nahm ihren Anfang in Verbindung mit dem Casa Bartholdy-Freskenzyklus in Rom in den Jahren 1816-1817. Zu Overbecks und Cornelius' führender Position siehe auch: Frank. German Romantic
Painting Redefined, 22-24. Painting Redefined, 22-24

6 Die Bezeichnung „neue altdeutsche Schule”, welche von Maydell verwendet wurde, weist auf die direkte Verbindung der nazarenischen Kunst mit den Vorbildern des (deutschen) Spätmittelalters und der frühen Renaissance hin.

27 Raffaels (1483-1520) frühes Schaffen und seine Madonnenbilder hob bereits einer der ersten Theoretiker des Romantizismus, Friedrich Schlegel (1772-1829), hervor.

28 Seit dem Jahr 1822 verkehrte Victor Orsel (1795-1850), der als Schüler des neuen Direktors der Académie française, Pierre-Narcisse Guérin, nach Rom gekommen war, intensiv mit Overbeck und den Nazarenern. Orsel ist bekannt als einer der ersten französischen Nazarener, doch Overbecks Einflüsse finden sich beispielsweise auch im Werk von Jean-Auguste-Dominique Ingres. Siehe: Howit Friedrich Overbeck: Sein Leben und Schaffen, 116

29 Schon in der Zeit des Studiums an der Wiener Akademie wurde es für die zukünftigen Nazarener ur Sitte, die Abende gemeinsam zu verbringen und die bearbeiteten Werke zu kritisieren. - Frank German Romantic Painting Redefined, 11. 
Anatomie vorgenommen wird. Der Vortrag der letztern ist mir übertragen, u. da ich d. nöthigen Bücher, Zeichnungen u. Kupferstiche dazu habe, so kann ich mich gehörig preparieren, $u$. habe selbst den meisten Nutzen davon. So ist mein Tag von Morgen bis Abend besetzt, u. dauert mir nur immer nicht lang genug. Mein [S. 4] großes Ziel steht mir beständig dabey vor Augen, u. Sie können denken, lieber Onkel, wie freudig ich einen Weg wandle, der mich demselben täglich näher bringt. Da noch keine andere Aussicht vor mir war, faßten Sie bester Onkel mich freundlich bey der Hand, $u$. wollten mich einen Weg leiten, den Sie als gut u. nützlich für mich erkannten, an eine Stelle, wo ich so weit meine Kräfte gingen, gleich Ihnen, meinen Mitbürgern zu Rath u. Hülfe stehen sollte. Was ich ihnen dafür schuldig bin, erkannte ich damals, u. werde es auch jetzt $u$. nie vergessen. Daß ich damals, obgleich ich mir es oft schwer abdringen mußte, doch treu an meiner Stelle zu seyn suchte, werden Sie vielleicht bemerkt haben, wenn ich gleich auch oft genug Ihren Wünschen nicht gehörig entsprochen haben mag. Ihr Beifall u. mein eigenes Bewustseyn, waren d. einzige Belohnung die ich erringen konnte, denn d. Arbeit war ja von der Art, daß nur Sie u. noch einige wenige, ihre Schwierigkeit kennen konnten. Diese zwei Jahre sind mir aber auch an mir selbst von großem Nutzen gewesen, drum glauben Sie mir gewiß, daß ich auch keine Stunde derselben, die ich mit d. Arbeit zubrachte, für verlohrene achte. Aber, liebster Onkel, vergeben Sie mir doch auch ja, daß ich, da sich auf einmal, ohne das geringeste absichtliche Zuthun auf meiner Seite, eine Bahn für mich öffnete, nach der ich mich ja Jahrelang gesehnt hatte, auf die meinen Gedanken in jedem müßigen Augenblicke schweiften, von der aus nur alle Dinge der Welt erst recht verständlich wurden, $u$. wo mir Mittel gebothen wurden, fest u. tief in das Leben zu greifen, daß ich diese Bahn einschlug so gewagt u. unsicher sie schien, $\mathrm{u}$. den sichern, stillen Weg verließ, auf den Sie mich geleitet hatten. Ich konnte nicht anders, lieber Onkel, ich konnte wirklich nicht anders, der Preis der mir geboten wurde, war zu schön u. herrlich. Ausdrücklich, u. mit wollem Bewustseyn hatte ich schon ganz resigniert auf ein Glück, daß mir unmöglich zu erlangen schien, aber solch eine Resignation kann wohl ruhig, nur nicht glücklich machen, u. ich hoffte gar nichts mehr für mich, lebte nur so einen Tag nach dem anderen ab, u. verschloß mir d. Zukunft so viel als möglich. Nicht jeder kann der Spaltung zwischen düsterem Beruf $\mathrm{u}$. innerem Trieb, so glücklich Herr werden; ich wenigstens vermochte es nicht. Wie hätte ich
Lechzender da nicht auf den hellen Born, der plötzlich vor mir aufsprudelte, zu eilen, $u$. meine Hütte dicht an ihn aufbauen sollen? Ich rechne daher sicher auf d. Güte, die Sie lieber Onkel, immer für mich gehabt haben, daß Sie mir meinen Abweg verzeihen, da Sie doch gewiß seyn können, daß es weder aus Nichtachtung Ihres Raths, noch aus Scheu vor Arbeit an sich, geschehen ist. Auch hier habe ich ja nicht aus Eigendünkel $u$. kitzelnder Lusf zugegriffen, sondern habe lange geprüft u. gewählt u. endlich doch andere, competente Richter entscheiden lassen. Böse sind Sie mir wohl gewiß nie darüber geworden, aber ich wünschte auch, daß Sie sogar mit meinem neuen wunderlichen Unterfangen zufrieden wären, das wäre mir erst das liebste. Vielleich finden Sie einmal ein müßiges Stündchen, mir mit ein paar solchen Worten, den Muth recht frisch zu machen. An Vater ${ }^{30}$ habe ich schon meinen Entschluss gemeldet, $\mathrm{u}$. will erst wenn ich seine Antwort habe, um den Abschied eingeben, wenn es nicht angehen sollte, daß er es in meinem Namen thäte, weshalb ich ihn auch gebeten. Die Verlängerung meines halbjährlichen Urlaubs, habe ich nicht erhalten, wohl aber das schriftliche Versprechen von Ewers, ${ }^{31}$ daß sie erfolgen solle, worauf ich auch in Bern, einen neuen Pass nach Italien bekam, der mich vor aller weitere Nachfrage bisher geschützt hat. - Möge, lieber Onkel, diese Erzählung meiner gegenwärtigen Lage, Ihnen lieb seyn, u. mir zur Erfüllung meiner Bitte mithelfen. Haben Sie d. Güte, die Tante ${ }^{32}$ recht sehr herzlich von mir zu grüßen, auch Gustchen ${ }^{33} u$. ihre lieben Mädchen, wenn die noch etwas von mir wissen. Bald hätte ich vergessen, meine Gratulation über d. glückliche Ankunft des jüngsten Kleinen, ${ }^{34}$ abzustatten. Da werde ich neue Bekanntschaft zu machen haben. Nun, leben

30 Reinhold Gottlieb von Maydell (1771-1846), estländischer Landrat, war Besitzer von Gut Stenhusen, Kirchspiel Goldenbeck, Kreis Wiek (Teenuse, Kullamaa khk, Läänemaa)

31 Der Rektor der Universität Dorpat, Gustav Ewers, war ebenfalls ein angeheirateter Verwandter von Maydells. Er war mit Dorothea von Maydell (1790-1864) vermählt, bei dieser handelte es sich um die Tochter des Cousins von Friedrich Ludwig von Maydells Großvater, des Besitzers von Gut (1737-1799). Dorspiel Goldenbeck, des Nachbarguts von Sten. Maydell (17 ( Werro, auf (Vaimela, Polva khk, Vorumaa).

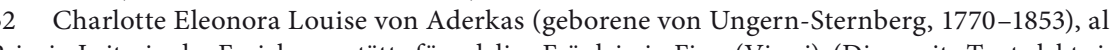
Priorin Leiterin der Erziehungsstätte für adelige Fräulein in Finn (Vinni). (Die zweite Tante lebte in Deutschland, die dritte Tante Juliane war bereits seit 1800 die Stiefmutter von Maydells.)

33 Margareta Augusta „Gustchen” von Forestier (geborene von Ungern-Sternberg, 1801-1872), die Tochter von Maydells Onkel Johann Friedrich Emmanuel von Ungern-Sternberg (1763-1825), lebte schon seit einiger Zeit bei ihrem Oheim und heiratete 1824 ihren Cousin Gustav Alexander vo

34 Moritz Rudolf von Ungern-Sternberg (1822-1890) 
Sie recht wohl, liebster Onkel, u. erhalten Sie Ihre bisherige Güte u. Liebe Ihrem Ihnen stets mit Dankbarkeit anhängenden Neffen

Ludwig v. Maydell

Moonika Teemus: The Letter of Friedrich Ludwig von Maydell from Rome (1823)

Keywords: Friedrich LudWig VON MAYdell; Ungern-Sternberg FAMily; Johann Friedrich Overbeck; Nazarene Movement; Biographies; Source PUBLICATION

\section{SUMMARY:}

The article studies a letter from Friedrich Ludwig von Maydell (17951846), the most renowned representative of Romanticism in Estonia, to his uncle Otto Christian Sigismund von Ungern-Sternberg (1778-1861), written in Rome at the beginning of the year 1823. It was the turning point in Maydell's life when he had decided to give up the studies in law at the University of Tartu and to devote himself to art. For this reason, Maydell like many of his contemporaries travelled to Rome. In his letter, now preserved in the National Archives in Tartu, Maydell describes his everyday life in Rome and the efforts he has made to "follow his true path". Additionally, it appears that it was the founder and leader of the Nazarene movement, Johann Friedrich Overbeck (1789-1869), who played an influential role in Maydell's decision about whether or not to become an artist.

CV:

Moonika Teemus (M.A.) is an Estonian art historian focusing mainly on the history of visual culture in Estonia in the period 1800-1850. Librarian and the curator of art collection at the University of Tartu Library. 